\title{
CONTROL OF PMDC MOTOR USING FUZZY PD CONTROLLER
}

\author{
Amerendra Reddy B. ${ }^{1}$, Rama Koti Reddy D.V. ${ }^{2}$, Srinivas Babu P.S. ${ }^{3}$, Anantha Babu P. \\ ${ }^{1}$ Andhra University College of Engineering, AP, India. \\ E-mail: ${ }^{1}$ amarendrareddybhimavarapu@rediffmail.com
}

\begin{abstract}
This paper presents the comparison of tracking performance of fuzzy PD controller with conventional controller to control PMDC Motor which employs only two fuzzy sets on the universe of discourse of each input variable and three fuzzy sets on the universe of discourse of output variable. Analytical structure of the simplest fuzzy PD controller is derived via triangular membership functions for fuzzification, Zadeh OR, mamdani's minimum inference method, and centroid method for defuzzification. The effectiveness of fuzzy PD controller over the conventional controller is shown for Permanent Magnet DC Motor with saturation nonlinearity by choosing gbell, trapezoidal, pi, Gaussian, triangular input fuzzy sets.
\end{abstract}

Keywords: Analytical Structure, Fuzzy control, Permanent Magnet DC Motor, PID control.

\section{INTRODUCTION}

Since the first successful application of the idea of the fuzzy sets (1) to the control of dynamic plant by mamdani and assilian (2), there has been considerable world wide interest in the subject of "Fuzzy Control System Engineering". It has been known that it is possible to control many complex systems effectively by human operators who have no knowledge of their underlying dynamics, while it is difficult to achieve the same with conventional controllers. It is this fact which has ultimately led to the prospective development of fuzzy control in a variety of applications (3), most of these applications have been based on the intuitive implementation of domain experts' experience.

"Analytical structure" we mean the mathematical expression of a fuzzy controller that represents precisely the fuzzy controller without any approximation. Note that this is never an issue for conventional control because the analytical structure of a conventional controller, linear or nonlinear, is always readily available for analysis and design. Thus the design goal is to design the controller structure and Parameters on the basis of the given system model so that resulting control system performance will meet user's performance specifications. For fuzzy control, in addition to this usual requirement, there exist few more major difficulties pertinent only to fuzzy control and irrelevant to conventional control. One of them is that the input-output structure of a fuzzy controller is usually mathematically unavailable after the controller is constructed, most fuzzy controllers are constructed via so called intelligent system approaches as opposed to the mathematical approaches exclusively used in conventional controller.

The fuzzy controller have been treated and used as black-box controllers without the analytical structure information, precise and effective mathematical analysis and design are very difficult to achieve. Hence the foremost issue is reveling the analytical structure is sensible in the context of conventional control theory. This is to say that merely deriving the structure is not useful enough and the structure must be represented in a form clearly understandable from control theory stand point. Once the structure is well understood, analytical issues can be explored using the well developed conventional control theory.

The present paper should be an attempt to understand what the fuzzy PD controller is and how well it can perform in comparison with the conventional PD controller. Here it has demonstrated that the fuzzy PD controller is better than the conventional one for nonlinear systems, this is not a defect of the conventional PD Controller. The conventional controller was designed for linear systems and this goal is achieved. But if the fuzzy PD controller can do the same job, at least as well as the conventional PD controller, for linear systems and if it can also handle some nonlinear systems at the same cost. The fuzzy PD controller developed has a new structure that is not a simple modification of the conventional version.

This paper organized as follows, section II describes the principal components of fuzzy PD controller. Section III describes fuzzification algorithm and fuzzy control rules, section IV describes fuzzy logic for evaluation of fuzzy control rules, section $V$ describes the defuzzification algorithm, and finally computer simulations are presented.

\section{CONFIGURATION OF THE FUZZY PD CONTROLLER}

The simple fuzzy PD controller consists of two inputs and one output. The input variables are error and change of error (rate, for short) of process output with respect to 
output setpoint. They are denoted as follows:

$$
\begin{aligned}
& e(n T)=s p(n T)-y(n t) \\
& r(n T)=e(n T)-e(n T-T)
\end{aligned}
$$

Where $\mathrm{n}$ is positive integer, $\mathrm{T}$ is sampling period and $s p(n T)$ is the setpoint. We denote $e(n T), r(n T)$ and $y(n T)$ as error, rate and process output, respectively. Each of the two input variables is fuzzified by two input fuzzy sets, named "positive" and "negative", and the output has three fuzzy sets, named as "positive", "zero" and "negative", whose membership functions are shown in below. In [-L, $L$ ], where $L$ is a design parameter, the membership functions are:

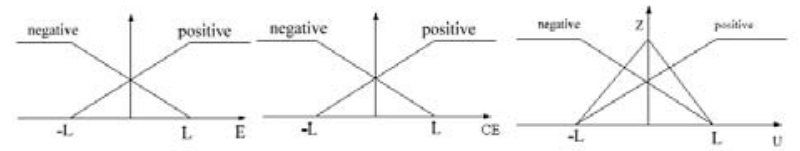

Fig. 1. shows the triangular membership functions of the error, rate and output

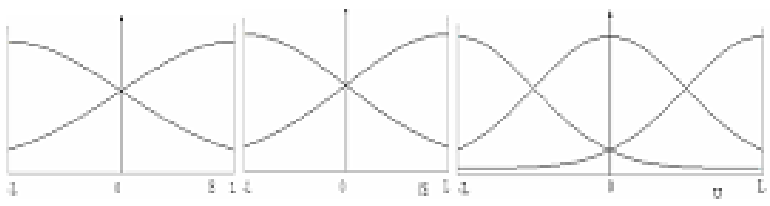

Fig. 2. Shows the gauss membership functions of the error and rate and the output

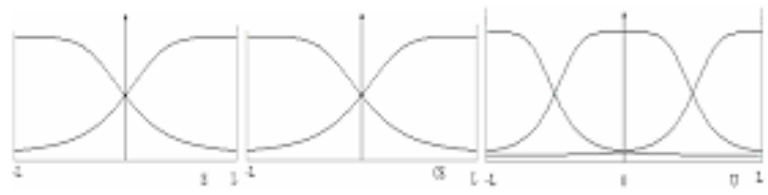

Fig. 3. Shows the gbell membership functions of the error and rate and the output

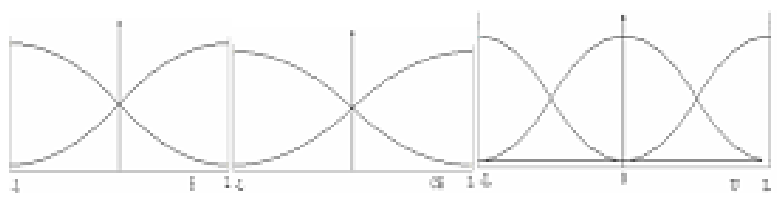

Fig. 4. Shows the Pi membership functions of the error and rate and the output

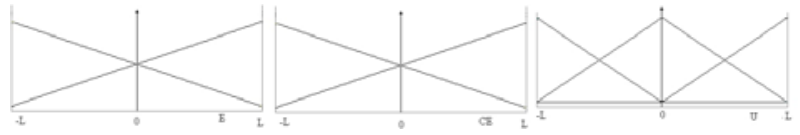

Fig. 5. Shows the trapezoidal membership functions of the error and rate and the output
Outside the $[-L, L]$, the memberships are either zero or one.

The components of fuzzy controller include:

A. The input scalers GE,GR and output scaler GU;

B. A fuzzification algorithm for scaled error, scaled rate and output;

C. The fuzzy control rules;

D. fuzzy logic used for evaluating the fuzzy control rules; and

E. Defuzzification algorithm used for getting crisp output from the fuzzy set "output".

\section{FUZZIFICATION ALGORITHM AND FUZZY CONTROL RULES}

In the fuzzification step, we employ two inputs: The fuzzy set "error" has two members, e.p (denotes error positive) and e.n (denotes error negative); and the fuzzy set "rate" has two members r.p (denotes rate positive) and r.n (denotes rate negative) with only one output which has three membership functions which are shown in fig1. The constant $L>0$ is chosen according to value ranges of the error, rate and output, which will be fixed after being determined.

The fuzzy PD controller uses the following four fuzzy control rules:

R1. If Error is positive AND rate is positive THEN output is zero.

R2. If Error is positive AND rate is negative THEN output is positive.

R3. If Error is negative AND rate is positive THEN output is negative.

R4. If Error is negative AND rate is negative THEN output is zero.

Here "output" is the fuzzy control action and AND is Zadeh's logical "AND" [1] defined by

$$
\mu_{\mathrm{A}} \operatorname{AND} \mu_{\mathrm{B}}=\min \left\{\mu_{\mathrm{A}} \cdot \mu_{\mathrm{B}}\right\}
$$

For any membership value and on the fuzzy subsets $A$ and $B$, respectively.

\section{FUZZY LOGIC FOR EVALUATION OF THE FUZZY CONTROL RULES}

The fuzzy logics with which we are concerned are those of Zadeh and of Lukasiewicz. If ${ }^{\circ} \mathrm{A}$ and ${ }^{\circ} \mathrm{B}$ represent the grades of membership of an object in fuzzy sets $A$ and $B$, respectively, then these logics are defined as: 
Zadeh logic:

$\operatorname{AND}\left({ }^{\circ},{ }_{A}^{\circ}\right)=\min \left({ }_{A}^{\circ},{ }_{B}^{\circ}\right)$

\section{DEFUZZIFICATION ALGORITHMS}

The nonlinear defuzzification procedure was used, which amounted to a normalization of the grades of membership of the members of the fuzzy set being defuzzified to sum of one. The defuzzified output of a fuzzy set is defined as

$$
\Delta u_{N L}=\frac{\text { Sum }[(\text { value of member }) \cdot(\text { membership of member })]}{\text { Sum(membership) }}
$$

Where is the defuzzified equivalent of the fuzzy set. The nonlinearity introduced into the defuzzification algorithm by the denominator.

In the rules Zadeh fuzzy logic AND is used and the centroid [6] defuzzifier is employed to calculate the output change of the fuzzy PD controller

$$
\Delta u(n T)=\frac{\sum \Delta u_{i} \cdot u_{r i}}{\sum u_{r i}}
$$

The new output of the fuzzy controller at is

$$
u(n T+T)=u(n T)+\Delta u(n T)
$$

\section{CASE STUDY}

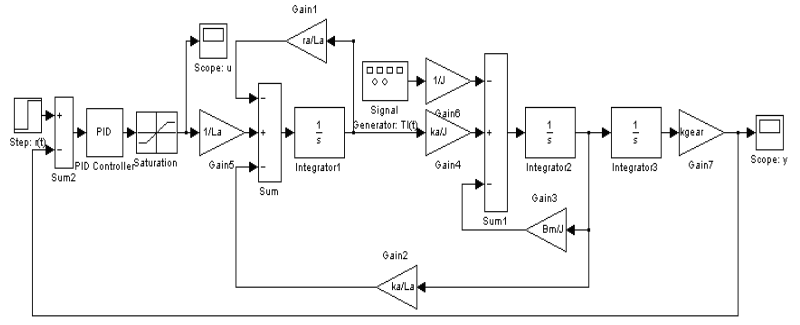

Fig. 6. P.M.D.C. Servo motor With Conventional PID Controller Having saturation nonlinearity

Consider the rotating table actuated by a permanent magnet DC motor, the motor is attached to the table through the gear with coefficient $k_{\text {gear }}$ Our goal is to guarantee the assigned angular displacement is a function of the rotor displacement, and $y(t)=k_{\text {gear }} \theta_{r}(t)$ is the output equation. to change the angular velocity and displacement, one regulates the armature voltage applied to the motor winding. The input of a servo motor is a voltage value and the output shaft of the servo motor is commanded to a particular angular position according to the input voltage. The applied voltage bounded as,. Servo motors are used in radio control airplanes to control the position of wing flaps and similar devices.

\section{SIMULATION RESULTS}

Computer simulation was used to determine the control performance of the fuzzy controller. The results of the fuzzy controller using the nonlinear defuzzification algorithm were compared to that of the conventional PD controller.

The responses by using the different membership functions are showed for the comparison purpose.
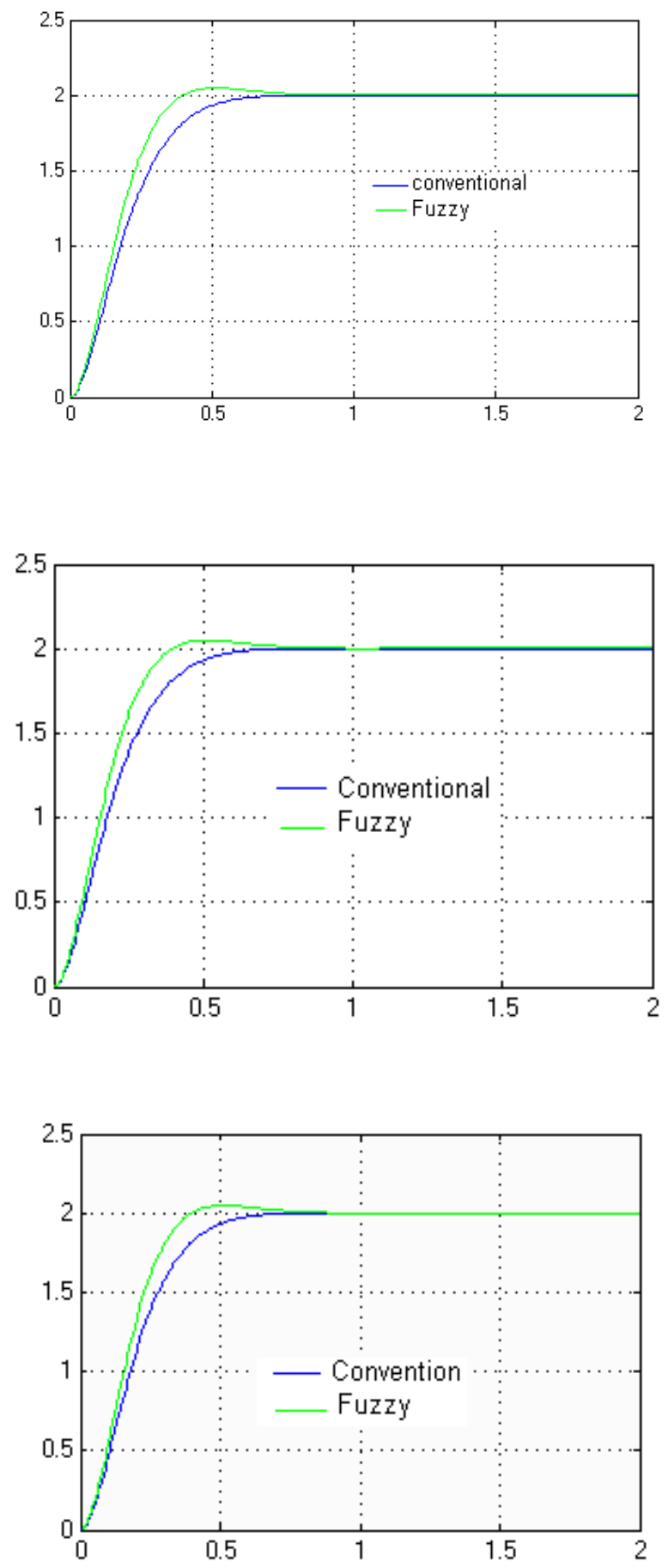

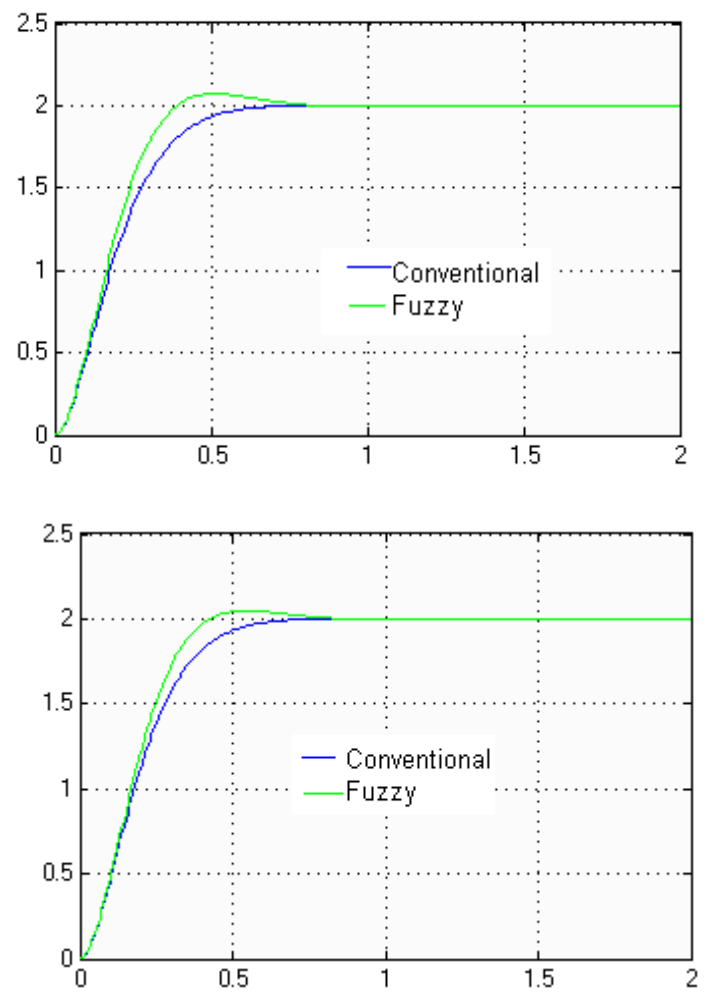

Fig. 7. the performance of the fuzzy PD controller for PMDC Motor without load and $\mathrm{T}=0$ by using Gbell,

Gaussian, Pi, Trapezoidal, Triangular input fuzzy sets and consolidated fuzzy membership functions.
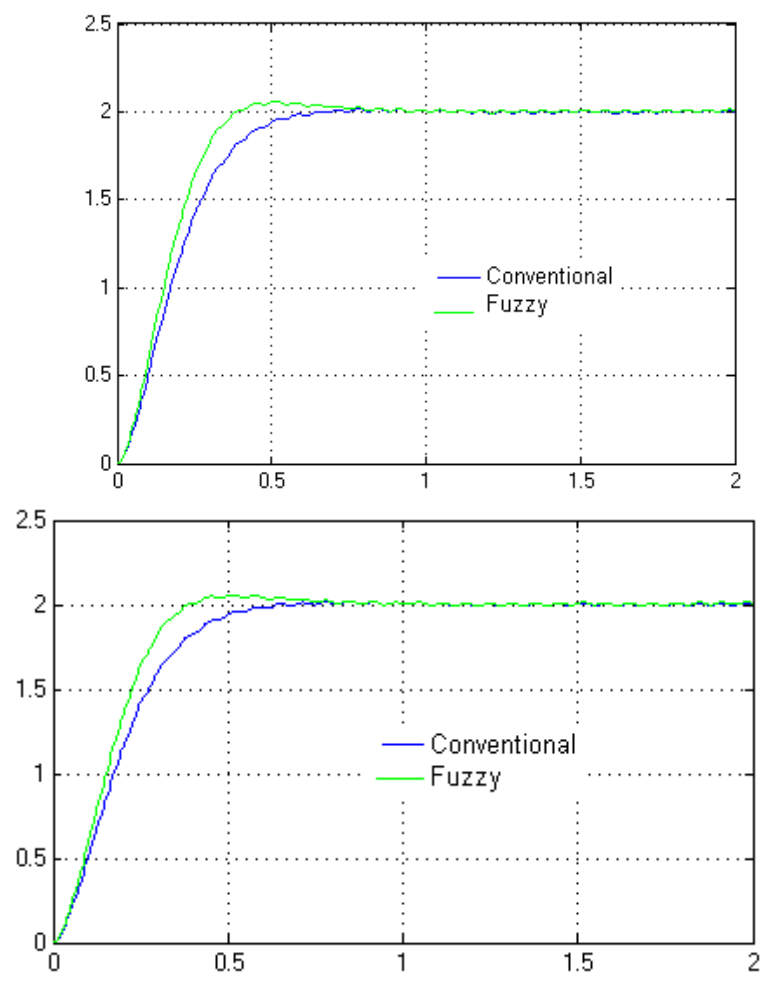
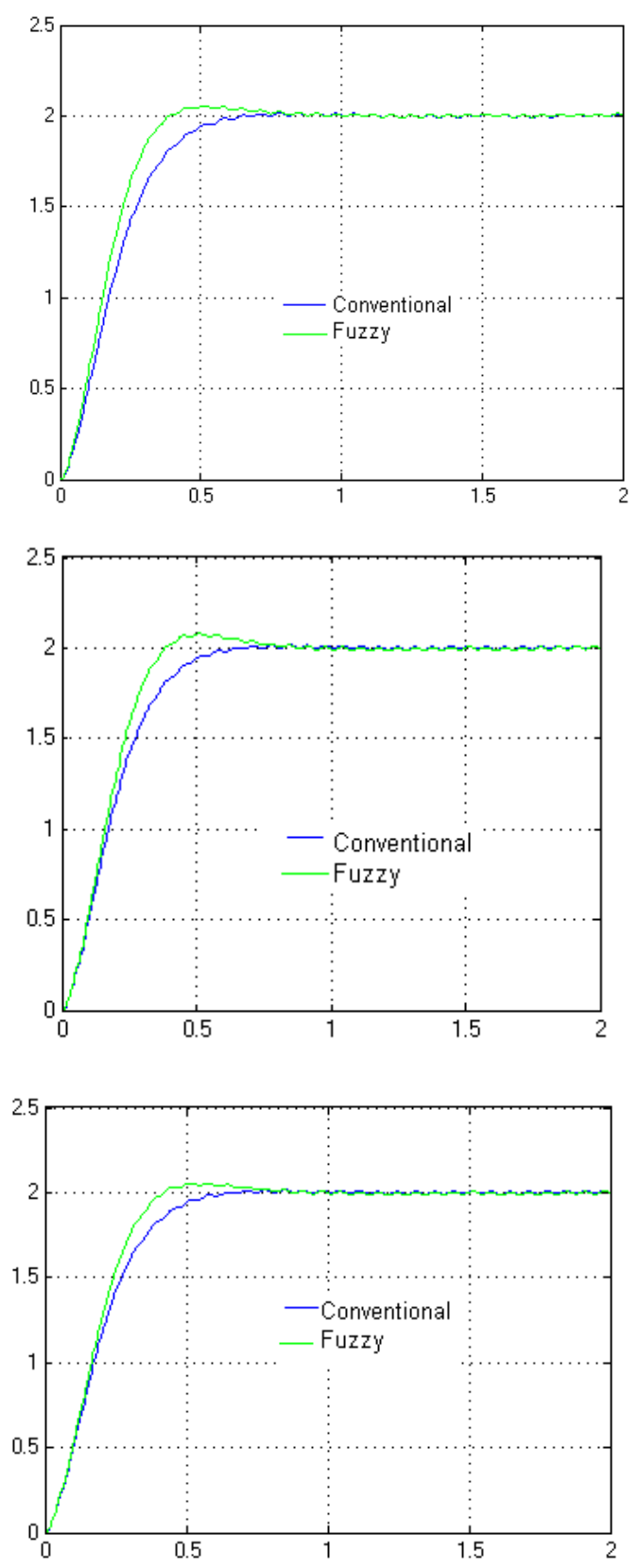

Fig. 8. the performance of the fuzzy PD controller for PMDC Motor with load disturbance and $\mathrm{T}=0$ by using Gbell, Gaussian, Pi, Trapezoidal, Triangular input fuzzy sets and consolidated fuzzy membership functions.

\section{CONCLUSION}

In this paper, we have described the basic steps that needed for tracking performance of the fuzzy PD controller. Here we have considered an application of PMDC Motor system which contains saturation nonlinearity for the tracking performance. Simulation results demonstrate the advantage of the fuzzy PD controller over the conventional one when the PMDC motor with saturation nonlinearity is subjected to with and 
without load disturbance. From the graphs it is clear that the tracking performances of fuzzy controllers are better than the conventional controller irrespective of the membership function used.

\section{REFERENCES}

[1] Zadeh, L. A, 1965, "Fuzzy sets", Inf. Control, 8, pp338-353.

[2] E.H Mamdani, S. Assilian,1974, "An experiment in linguistic synthesis with a fuzzy logic controller", internat. J. Man machine stud. 7 (1). Pp1-13.

[3] Sugeno, M, 1985, "Industrial applications of fuzzy control", Amsterdam: North-Holland.

[4] Siler, W. and Ying, 1989, H "Fuzzy Control Theory: The linear case", Fuzzy Sets and Systems, Vol. 33. pp. 275-290.

[5] Siller, W. Ying. H and J. J. Buckley, 1988, "Fuzzy control theory: a nonlinear case", Proc. Of NASA's conference on Artificial Neural systems and fuzzy logic, Houston, U.S.A.

[6] Ying. H and Siler, W. and Buckley, J.J , 1990, " Fuzzy Control Theory: A Nonlinear case", Automatica, Vol. 26(3), pp. 513-520.

[7] C. C. Lee, 1990, "Fuzzy logic in control systems: Fuzzy logic controller - part 1 and 2",IEEE Trans. Systems, Man Cybernet. SMC-20(2). pp. 404-435.

[8] Ying.H, 1993, "The simplest fuzzy controllers using different inference methods are different nonlinear Proportional-integral controllers with variable gains", Automatica. Vol. 29(6). pp. 1579-1589.
[9] Ying. H, 1994, "analytical structure of fuzzy controllers with linear control rules", Inf. Sci., vol. 81, pp 213-227.

[10] G. Chen and Ying, 1993, H "Stability analysis of nonlinear fuzzy PI controller systems ", Proc. ${ }^{\text {rd }}$ Int. conf. on Fuzzy logic applications, pp. 128-133.

[11] J. Lee, 1993, "On methods for improving performance of PI type fuzzy logic controllers", IEEE Trans. Fuzzy systems, vol. 1, pp. 298-301.

[12] H. A. Malki, H. Li and G. Chen,1994, "New design and stability analysis of fuzzy proportionalderivative control systems", IEEE Trans. Fuzzy systems 2 (4), pp. 245-254.

[13] Sergey Edward Lyshevski, "Control Systems Theory with Engineering Applications", Springer International Edition.

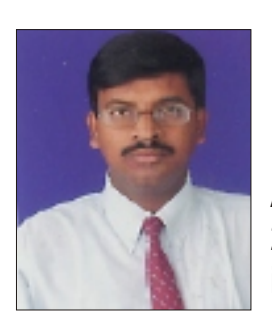

B. Amarendra Reddy graduated from Bapatla Engineering College, Bapatla, Nagarjuna University, in1999 with $\mathrm{EEE}$, and ME in Control Systems from Andhra University, 2001. From 2001 to 2006 he worked as Assistant Professor in Department of EEE, LBCE, Mylavaram, Andhra Pradesh.

Presently he is working as Assistant Professor in Department of Electrical Engineering, AU College of Engineering, Andhra University.His area of research include Control systems, Fuzzy Logic etc. 